\title{
Ann Arbor Clinical Stage I
}

National Cancer Institute

\section{Source}

National Cancer Institute. Ann Arbor Clinical Stage I. NCI Thesaurus. Code C125472.

A clinical stage that refers to lymphoma involving a single lymph node region or a single extranodal organ (le). 